\title{
Sedentary Time is Associated with Cardiometabolic Diseases in A Large Japanese Population: A Cross-Sectional Study
}

\author{
Teruhide Koyama $^{1}$, Nagato Kuriyama ${ }^{1}$, Etsuko Ozaki ${ }^{1}$, Satomi Tomida ${ }^{1,2}$, Ritei Uehara ${ }^{1}$, Yuichiro Nishida ${ }^{3}$, \\ Chisato Shimanoe ${ }^{4}$, Asahi Hishida ${ }^{5}$, Takashi Tamura ${ }^{5}$, Mineko Tsukamoto ${ }^{5}$, Yuka Kadomatsu ${ }^{5}$, Isao Oze ${ }^{6}$, \\ Keitaro Matsuo ${ }^{6,7}$, Haruo Mikami ${ }^{8}$, Yohko Nakamura ${ }^{8}$, Rie Ibusuki ${ }^{9}$, Toshiro Takezaki ${ }^{9}$, Sadao Suzuki ${ }^{10}$, \\ Takeshi Nishiyama $^{10}$, Kiyonori Kuriki ${ }^{11}$, Naoyuki Takashima ${ }^{12,}{ }^{13}$, Aya Kadota ${ }^{13}$, Hirokazu Uemura ${ }^{14}$, \\ Sakurako Katsuura-Kamano ${ }^{14}$, Hiroaki Ikezaki ${ }^{15}$, Masayuki Murata ${ }^{15}$, Kenji Takeuchi ${ }^{5}$ and Kenji Wakai ${ }^{5}$ \\ for the Japan Multi-institutional Collaborative Cohort (J-MICC) Study Group
}

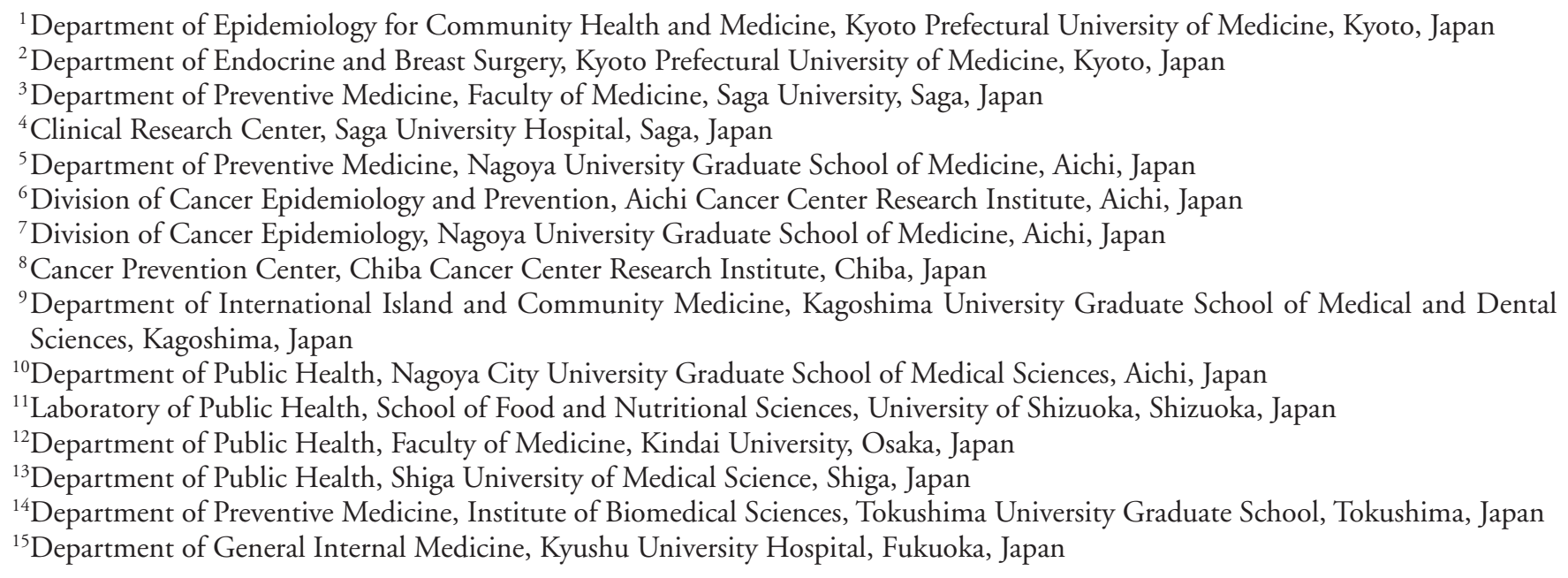

Aim: Accumulating evidence reveals that sedentary behavior is associated with mortality and cardiometabolic disease; however, there are potential age and sex differences in sedentary behavior and health outcomes that have not been adequately addressed. This study aimed to determine the association of sedentary behavior with cardiometabolic diseases such as hypertension, dyslipidemia, diabetes mellitus, and its risk factors in a large Japanese population according to age and sex.

Methods: Using data from the Japan Multi-Institutional Collaborative Cohort Study obtained from baseline surveys, data of 62,754 participants $(27,930$ males, 34,824 females) were analyzed. This study uses a cross-sectional design and self-administered questionnaires to evaluate sedentary time and anamnesis. For the logistic regression analysis, sedentary time $<5 \mathrm{~h} /$ day was used as the reference and then adjusted for age, research areas, leisure-time metabolic equivalents, and alcohol and smoking status. From the analysis of anthropometric and blood examinations, 35,973 participants (17,109 males, 18,864 females) were analyzed.

Results: For hypertension and diabetes, sedentary time was associated with a significantly higher proportion of male participants. Both sexes were associated with a significantly higher proportion of participants with dyslipidemia. Participants who had longer sedentary time tended to have increased levels of blood pressure, triglycerides, and non-high-density lipoprotein cholesterol (HDL-C), and decreased levels of HDL-C, especially in the 60-69 years group.

Conclusions: Independent of leisure-time physical activity, sedentary time was associated with cardiometabolic diseases in a large Japanese population classified by age and sex. Our findings indicate that regularly interrupting and replacing sedentary time may contribute to better physical health-related quality of life. 


\section{Introduction}

Accumulating evidence reveals that sedentary (sitting or reclining posture) behavior is associated with negative health connotations ${ }^{1)}$, including cardiovascular-specific and overall mortality ${ }^{2)}$. Similarly, studies have demonstrated a relationship between sedentary behavior and the development of metabolic disease (e.g., obesity, metabolic syndrome, and type 2 diabetes mellitus) ${ }^{3}$. More specifically, these studies report an association between prolonged periods of sedentary behavior and all-cause morbidity and mortality, which cannot be simply explained by differences in engagement in low-, moderate-, or vigorous-intensity physical activity ${ }^{4}$. Sedentary time is associated with an increased risk of mortality and cardiometabolic disease, although there are potential age and sex differences in sedentary behavior and health outcomes that have not been adequately addressed ${ }^{5)}$.

In Japan, some studies showed an association between sedentary time and erectile dysfunction among patients with type 2 diabetes mellitus ${ }^{6}$, kidney function decline ${ }^{7)}$, chronic obstructive pulmonary disease $^{8)}$, coronary artery disease ${ }^{9)}$, and all-cause mortality ${ }^{10)}$. However, in Japan, few studies have examined the association between sedentary time and cardiovascular risk according to age and sex.

\section{Aim}

This study aimed to determine the association of sedentary behavior with cardiometabolic diseases such as hypertension, dyslipidemia, diabetes mellitus, and its risk factors in a large Japanese population according to age and sex.

\section{Methods}

\section{Study Participants}

In this study, we evaluated participant data collected during the Japan Multi-Institutional Collaborative Cohort (J-MICC) Study ${ }^{11)}$ from baseline surveys using cross-sectional data. The cohort study evaluated the general Japanese population using genetic and clinical data to detect and confirm gene-environment interactions related to lifestyle-associated diseases. The study participants were 35-69 years old and were enrolled after responding to study announcements in 13 research areas, attending health check-up examina-

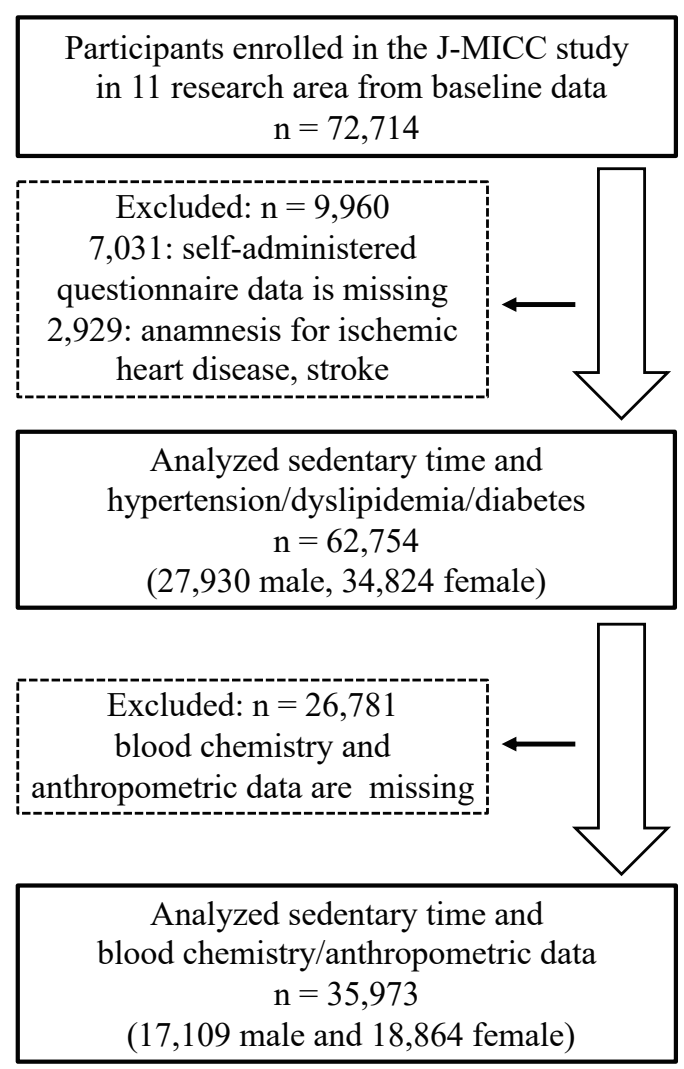

Fig. 1. Flow chart of the study participants

tions that were commissioned by their local governments, visiting local health check-up centers, or visiting a cancer hospital. Fig. 1 shows the flow chart of the study participants. Of the 13 research sites, two did not collect data on daily life activities, including sitting time from the participants. Excluding the participants in these two research sites, 72,714 participants were initially included in the current study (the dataset is ver. 20190729). Among the 72,714 participants, we excluded a total of 9,960 participants who lacked self-administered questionnaire data as follows: 478 without data on history of hypertension, dyslipidemia, or diabetes; 5,198 without data on smoking and drinking status or daily physical activity times including sitting time; 1,306 without data on medical history of ischemic heart disease and stroke; and 49 without data on drug treatment for hypertension, dyslipidemia, or diabetes. We also excluded 2,929 participants with a medical history of ischemic heart disease and stroke. Data for a total of 62,754 participants $(27,930$ males, 34,824 females) were analyzed for the associa-

Address for correspondence: Teruhide Koyama, Department of Epidemiology for Community Health and Medicine, Kyoto Prefectural University of Medicine 465 Kajii-cho, Kamigyo-ku, Kyoto 602-8566, Japan E-mail: tkoyama@koto.kpu-m.ac.jp

Received: November 24, 2019 Accepted for publication: February 11, 2020

Copyright@2020 Japan Atherosclerosis Society

This article is distributed under the terms of the latest version of CC BY-NC-SA defined by the Creative Commons Attribution License. 
tion of sedentary time with hypertension, dyslipidemia, and diabetes.

Furthermore, we analyzed participants from seven research sites who underwent anthropometric and blood examinations. We excluded 26,781 participants with missing data for body mass index (BMI), blood pressure (systolic blood pressure $[\mathrm{SBP}]$ and diastolic blood pressure $[\mathrm{DBP}])$, and biochemical measurements, including serum triglyceride (TG), highdensity lipoprotein cholesterol (HDL-C), non-HDL$\mathrm{C}$, and glycated hemoglobin (HbA1c). Finally, a total of 35,973 participants $(17,109$ males and 18,864 females) were included in the analysis of sedentary time.

All study participants gave written informed consent. The study protocol was approved by the Ethics Committees at Aichi Cancer Center, the Nagoya University Graduate School of Medicine (IRB No. 939-13), and other institutions participating in the J-MICC study. This study was conducted according to the principles expressed in the World Medical Association Declaration of Helsinki.

\section{Lifestyle and Blood Biochemistry Data}

In this study, we evaluated the lifestyle and medical information obtained through self-administered questionnaires (smoking and drinking status and physical activity, including sitting time). Physical activity was determined using a format similar to a short format of the International Physical Activity Questionnaire (IPAQ) ${ }^{12)}$. Leisure-time physical activity was assessed in terms of metabolic equivalents (LTMETs), as previously reported ${ }^{13}{ }^{14}$. In brief, METshours per day of leisure-time activity was estimated by multiplying the reported daily time that was spent in each activity by the relevant MET intensity. The duration of sitting time was classified into one of the following eight categories: none, $<1 \mathrm{~h} /$ day, 1 to $<3 \mathrm{~h} /$ day, 3 to $<5 \mathrm{~h} /$ day, 5 to $<7 \mathrm{~h} /$ day, 7 to $<9$ h/day, 9 to $<11 \mathrm{~h} /$ day, and $\geq 11 \mathrm{~h} /$ day. Sitting time was then categorized based on the quartile value: $5 \mathrm{~h} /$ day, $5-<$ $7 \mathrm{~h} /$ day, $7-<9 \mathrm{~h} /$ day, or $\geq 9 \mathrm{~h} /$ day. BMI was calculated as weight $(\mathrm{kg})$ divided by the square of height $\left(\mathrm{m}^{2}\right)$. Anamnesis and medication history were assessed using self-administered questionnaires. Hypertension, dyslipidemia, and diabetes were defined as the presence or absence of anamnesis and/or current use of medication. In addition, blood chemistry data (serum levels of TG, total cholesterol, HDL-C, non-HDL-C, and $\mathrm{HbA1c}$ ) and anthropometric data were obtained from health check-ups performed in the research areas. Laboratories, in each research area, analyzed the serum samples.

\section{Statistical Analyses}

Continuous variables are expressed as means, and categorical data are expressed as sums and percentages. We classified sedentary time into four groups according to sitting time ( $<5 \mathrm{~h}, 5$ to $<7 \mathrm{~h}, 7$ to $<9 \mathrm{~h}$, and $\geq 9 \mathrm{~h}$ ) and analyzed each group for sex and age in years (35-49, 50-59, and 60-69 years). Odds ratios (OR) and 95\% confidence intervals (CI) were calculated using logistic regression analyses to evaluate the associations of sedentary time with the prevalence of hypertension, dyslipidemia, and diabetes. Sedentary time $<5 \mathrm{~h}$ was used as a reference. Multiple regression analysis was performed to assess the sitting time $(<5$ $\mathrm{h}, 5$ to $<7 \mathrm{~h}, 7$ to $<9 \mathrm{~h}$, and $\geq 9 \mathrm{~h}$ ) influence on variables of cardiometabolic risk factors. The following factors were considered as independent variables: age, research areas, LT-METs, and drinking and smoking status (never, former, and current). Tests for linear trends (e.g., $P$ trend tests) were conducted by including four groups according to sitting time as ordinal variables. This provided a test of significance for the hypothesis that as the amount of sedentary time increases, the risk of cardiometabolic diseases tends to increase. All statistical tests were two-tailed, and differences with a $p$-value $<0.05$ were considered statistically significant. JMP 13 software (SAS Institute Inc., Cary, NC) was used for all statistical analyses.

\section{Results}

\section{Participant Characteristics}

Table 1 presents the participants' characteristics, including drinking and smoking status, anamnesis of hypertension/dyslipidemia/diabetes, and distribution of age and sex differences according to sedentary time. Among the 27,930 participants included in the current analysis for male sex, 9,529 (34.1\%), 5,458 (19.5\%), 4,491 (16.1\%), and 8,452 (30.3\%) spent sedentary time $<5 \mathrm{~h}, 5$ to $<7 \mathrm{~h}, 7$ to $<9 \mathrm{~h}$, and $\geq 9$ h per day, respectively. Similarly, among the 34,824 participants included in the current analysis for female sex, 14,121 (40.5\%), 8,727 (25.1\%), 5,715 (16.4\%), and $6,261(18.0 \%)$ spent sedentary time $<5 \mathrm{~h}, 5$ to $<7 \mathrm{~h}, 7$ to $<9 \mathrm{~h}$, and $\geq 9 \mathrm{~h}$ per day, respectively.

With increasing age, the proportion of sedentary time decreased from $34.5 \%$ to $26.0 \%$ for males in the 35-49 years old group vs. the 60-69 years old group and decreased from $21.8 \%$ to $15.0 \%$ for females.

The multivariate-adjusted OR for anamnesis of hypertension, dyslipidemia, and diabetes, according to sedentary time, is shown in Table 2. For the logistic regression analysis, sedentary time $<5 \mathrm{~h}$ /day was used as the reference and then adjusted for age, research areas, LT-METs, and alcohol and smoking status. For 
Table 1. Characteristics of participants according to sedentary time

\begin{tabular}{|c|c|c|c|c|c|c|c|c|}
\hline & \multicolumn{8}{|l|}{ Male } \\
\hline & \multicolumn{2}{|c|}{$\begin{array}{c}<5 \mathrm{~h} \\
n=9,529\end{array}$} & \multicolumn{2}{|c|}{$\begin{array}{c}5 \text { to }<7 \mathrm{~h} \\
n=5,458\end{array}$} & \multicolumn{2}{|c|}{$\begin{array}{c}7 \text { to }<9 \text { h } \\
n=4,491\end{array}$} & \multicolumn{2}{|c|}{$\begin{array}{c}\geq 9 \mathrm{~h} \\
n=8,452\end{array}$} \\
\hline & $n /$ mean & $(\%) / S D$ & $n /$ mean & $(\%) / S D$ & $n /$ mean & $(\%) / S D$ & $n /$ mean & $(\%) / S D$ \\
\hline \multicolumn{9}{|l|}{ years } \\
\hline $35-49$ & 2,616 & $31.5 \%$ & 1,480 & $17.8 \%$ & 1,340 & $16.1 \%$ & 2,868 & $34.5 \%$ \\
\hline $50-59$ & 2,926 & $33.9 \%$ & 1,612 & $18.7 \%$ & 1,367 & $15.8 \%$ & 2,727 & $31.6 \%$ \\
\hline $60-69$ & 3,987 & $36.3 \%$ & 2,366 & $21.5 \%$ & 1,784 & $16.2 \%$ & 2,857 & $26.0 \%$ \\
\hline \multicolumn{9}{|l|}{$35-49$ years } \\
\hline \multicolumn{9}{|l|}{ Drinking status } \\
\hline Current & 1,944 & $74.3 \%$ & 1,158 & $78.2 \%$ & 1,045 & $78.0 \%$ & 2,180 & $76.0 \%$ \\
\hline Former & 46 & $1.8 \%$ & 25 & $1.7 \%$ & 16 & $1.2 \%$ & 48 & $1.7 \%$ \\
\hline Never & 626 & $23.9 \%$ & 297 & $20.1 \%$ & 279 & $20.8 \%$ & 640 & $22.3 \%$ \\
\hline \multicolumn{9}{|l|}{ Smoking status } \\
\hline Current & 1,100 & $42.0 \%$ & 522 & $35.3 \%$ & 430 & $32.1 \%$ & 844 & $29.4 \%$ \\
\hline Former & 751 & $28.7 \%$ & 465 & $31.4 \%$ & 456 & $34.0 \%$ & 934 & $32.6 \%$ \\
\hline Never & 765 & $29.2 \%$ & 493 & $33.3 \%$ & 454 & $33.9 \%$ & 1,090 & $38.0 \%$ \\
\hline Hypertension & 224 & $8.6 \%$ & 133 & $9.0 \%$ & 129 & $9.6 \%$ & 269 & $9.4 \%$ \\
\hline Dyslipidemia & 279 & $10.7 \%$ & 178 & $12.0 \%$ & 182 & $13.6 \%$ & 409 & $14.3 \%$ \\
\hline Diabetes & 78 & $3.0 \%$ & 41 & $2.8 \%$ & 48 & $3.6 \%$ & 85 & $3.0 \%$ \\
\hline LT-METs (METs・hrs/day) & 1.90 & 3.43 & 1.83 & 3.00 & 1.78 & 2.73 & 1.49 & 2.20 \\
\hline \multicolumn{9}{|l|}{$50-59$ years } \\
\hline \multicolumn{9}{|l|}{ Drinking status } \\
\hline Current & 2,285 & $78.1 \%$ & 1,255 & $77.9 \%$ & 1,078 & $78.9 \%$ & 2,154 & $79.0 \%$ \\
\hline Former & 62 & $2.1 \%$ & 48 & $3.0 \%$ & 35 & $2.6 \%$ & 74 & $2.7 \%$ \\
\hline Never & 579 & $19.8 \%$ & 309 & $19.2 \%$ & 254 & $18.6 \%$ & 499 & $18.3 \%$ \\
\hline \multicolumn{9}{|l|}{ Smoking status } \\
\hline Current & 1,041 & $35.6 \%$ & 547 & $33.9 \%$ & 413 & $30.2 \%$ & 818 & $30.0 \%$ \\
\hline Former & 1,114 & $38.1 \%$ & 683 & $42.4 \%$ & 585 & $42.8 \%$ & 1,199 & $44.0 \%$ \\
\hline Never & 771 & $26.3 \%$ & 382 & $23.7 \%$ & 369 & $27.0 \%$ & 710 & $26.0 \%$ \\
\hline Hypertension & 640 & $21.9 \%$ & 373 & $23.1 \%$ & 343 & $25.1 \%$ & 631 & $23.1 \%$ \\
\hline Dyslipidemia & 449 & $15.3 \%$ & 320 & $19.9 \%$ & 331 & $24.2 \%$ & 671 & $24.6 \%$ \\
\hline Diabetes & 221 & $7.6 \%$ & 134 & $8.3 \%$ & 108 & $7.9 \%$ & 239 & $8.8 \%$ \\
\hline LT-METs (METs • hrs/day) & 1.76 & 3.14 & 1.99 & 3.11 & 1.84 & 2.61 & 1.66 & 2.32 \\
\hline \multicolumn{9}{|l|}{ 60-69 years } \\
\hline \multicolumn{9}{|l|}{ Drinking status } \\
\hline Current & 3,113 & $78.1 \%$ & 1,812 & $76.6 \%$ & 1,345 & $75.4 \%$ & 2,059 & $72.1 \%$ \\
\hline Former & 128 & $3.2 \%$ & 111 & $4.7 \%$ & 84 & $4.7 \%$ & 176 & $6.2 \%$ \\
\hline Never & 746 & $18.7 \%$ & 443 & $18.7 \%$ & 355 & $19.9 \%$ & 622 & $21.8 \%$ \\
\hline \multicolumn{9}{|l|}{ Smoking status } \\
\hline Current & 925 & $23.2 \%$ & 530 & $22.4 \%$ & 390 & $21.9 \%$ & 650 & $22.8 \%$ \\
\hline Former & 1,826 & $45.8 \%$ & 1,142 & $48.3 \%$ & 922 & $51.7 \%$ & 1,504 & $52.6 \%$ \\
\hline Never & 1,236 & $31.0 \%$ & 694 & $29.3 \%$ & 472 & $26.5 \%$ & 703 & $24.6 \%$ \\
\hline Hypertension & 1,328 & $33.3 \%$ & 834 & $35.2 \%$ & 598 & $33.5 \%$ & 1,005 & $35.2 \%$ \\
\hline Dyslipidemia & 712 & $17.9 \%$ & 519 & $21.9 \%$ & 407 & $22.8 \%$ & 678 & $23.7 \%$ \\
\hline Diabetes & 486 & $12.2 \%$ & 301 & $12.7 \%$ & 245 & $13.7 \%$ & 383 & $13.4 \%$ \\
\hline LT-METs (METs・hrs/day) & 3.25 & 4.67 & 3.31 & 3.92 & 3.02 & 3.53 & 2.47 & 3.25 \\
\hline
\end{tabular}


(Cont. Table 1)

\begin{tabular}{|c|c|c|c|c|c|c|c|c|}
\hline & \multicolumn{8}{|l|}{ Female } \\
\hline & \multicolumn{2}{|c|}{$\begin{array}{c}<5 \mathrm{~h} \\
n=14,121\end{array}$} & \multicolumn{2}{|c|}{$\begin{array}{c}5 \text { to }<7 \mathrm{~h} \\
n=8,727\end{array}$} & \multicolumn{2}{|c|}{$\begin{array}{l}7 \text { to }<9 h \\
n=5,715\end{array}$} & \multicolumn{2}{|c|}{$\begin{aligned} & \geq 9 \mathrm{~h} \\
n & =6,261\end{aligned}$} \\
\hline & $n /$ mean & $(\%) / S D$ & $n /$ mean & $(\%) / S D$ & $n /$ mean & $(\%) / S D$ & $n /$ mean & $(\%) / \mathrm{SD}$ \\
\hline \multicolumn{9}{|l|}{ years } \\
\hline $35-49$ & 4,952 & $39.9 \%$ & 2,750 & $22.1 \%$ & 2,007 & $16.2 \%$ & 2,709 & $21.8 \%$ \\
\hline $50-59$ & 4,605 & $42.3 \%$ & 2,745 & $25.2 \%$ & 1,723 & $15.8 \%$ & 1,823 & $16.7 \%$ \\
\hline $60-69$ & 4,564 & $39.7 \%$ & 3,232 & $28.1 \%$ & 1,985 & $17.2 \%$ & 1,729 & $15.0 \%$ \\
\hline \multicolumn{9}{|l|}{$35-49$ years } \\
\hline \multicolumn{9}{|l|}{ Drinking status } \\
\hline Current & 2,358 & $47.6 \%$ & 1,379 & $50.1 \%$ & 995 & $49.6 \%$ & 1,391 & $51.3 \%$ \\
\hline Former & 118 & $2.4 \%$ & 49 & $1.8 \%$ & 42 & $2.1 \%$ & 80 & $3.0 \%$ \\
\hline Never & 2,476 & $50.0 \%$ & 1,322 & $48.1 \%$ & 970 & $48.3 \%$ & 1,238 & $45.7 \%$ \\
\hline \multicolumn{9}{|l|}{ Smoking status } \\
\hline Current & 516 & $10.4 \%$ & 232 & $8.4 \%$ & 165 & $8.2 \%$ & 292 & $10.8 \%$ \\
\hline Former & 541 & $10.9 \%$ & 306 & $11.1 \%$ & 211 & $10.5 \%$ & 305 & $11.3 \%$ \\
\hline Never & 3,895 & $78.7 \%$ & 2,212 & $80.4 \%$ & 1,631 & $81.3 \%$ & 2,112 & $78.0 \%$ \\
\hline Hypertension & 188 & $3.8 \%$ & 93 & $3.4 \%$ & 87 & $4.3 \%$ & 96 & $3.5 \%$ \\
\hline Dyslipidemia & 223 & $4.5 \%$ & 122 & $4.4 \%$ & 84 & $4.2 \%$ & 153 & $5.6 \%$ \\
\hline Diabetes & 36 & $0.7 \%$ & 25 & $0.9 \%$ & 18 & $0.9 \%$ & 23 & $0.8 \%$ \\
\hline LT-METs (METs • hrs/day) & 1.54 & 2.74 & 1.51 & 2.51 & 1.33 & 2.18 & 1.12 & 1.86 \\
\hline \multicolumn{9}{|l|}{$50-59$ years } \\
\hline \multicolumn{9}{|l|}{ Drinking status } \\
\hline Current & 1,836 & $39.9 \%$ & 1,093 & $39.8 \%$ & 728 & $42.3 \%$ & 765 & $42.0 \%$ \\
\hline Former & 64 & $1.4 \%$ & 44 & $1.6 \%$ & 27 & $1.6 \%$ & 33 & $1.8 \%$ \\
\hline Never & 2,705 & $58.7 \%$ & 1,608 & $58.6 \%$ & 968 & $56.2 \%$ & 1,025 & $56.2 \%$ \\
\hline \multicolumn{9}{|l|}{ Smoking status } \\
\hline Current & 410 & $8.9 \%$ & 180 & $6.6 \%$ & 107 & $6.2 \%$ & 150 & $8.2 \%$ \\
\hline Former & 331 & $7.2 \%$ & 211 & $7.7 \%$ & 129 & $7.5 \%$ & 162 & $8.9 \%$ \\
\hline Never & 3,864 & $83.9 \%$ & 2,354 & $85.8 \%$ & 1,487 & $86.3 \%$ & 1,511 & $82.9 \%$ \\
\hline Hypertension & 697 & $15.1 \%$ & 421 & $15.3 \%$ & 227 & $13.2 \%$ & 244 & $13.4 \%$ \\
\hline Dyslipidemia & 753 & $16.4 \%$ & 496 & $18.1 \%$ & 305 & $17.7 \%$ & 352 & $19.3 \%$ \\
\hline Diabetes & 151 & $3.3 \%$ & 86 & $3.1 \%$ & 45 & $2.6 \%$ & 62 & $3.4 \%$ \\
\hline LT-METs (METs • hrs/day) & 1.85 & 2.88 & 2.00 & 2.86 & 1.65 & 2.24 & 1.38 & 2.11 \\
\hline \multicolumn{9}{|l|}{ 60-69 years } \\
\hline \multicolumn{9}{|l|}{ Drinking status } \\
\hline Current & 1,372 & $30.1 \%$ & 1,071 & $33.1 \%$ & 669 & $33.7 \%$ & 577 & $33.4 \%$ \\
\hline Former & 51 & $1.1 \%$ & 53 & $1.6 \%$ & 37 & $1.9 \%$ & 44 & $2.5 \%$ \\
\hline Never & 3,141 & $68.8 \%$ & 2,108 & $65.2 \%$ & 1,279 & $64.4 \%$ & 1,108 & $64.1 \%$ \\
\hline \multicolumn{9}{|l|}{ Smoking status } \\
\hline Current & 166 & $3.6 \%$ & 99 & $3.1 \%$ & 79 & $4.0 \%$ & 79 & $4.6 \%$ \\
\hline Former & 194 & $4.3 \%$ & 142 & $4.4 \%$ & 105 & $5.3 \%$ & 108 & $6.2 \%$ \\
\hline Never & 4,204 & $92.1 \%$ & 2,991 & $92.5 \%$ & 1,801 & $90.7 \%$ & 1,542 & $89.2 \%$ \\
\hline Hypertension & 1,155 & $25.3 \%$ & 846 & $26.2 \%$ & 535 & $27.0 \%$ & 470 & $27.2 \%$ \\
\hline Dyslipidemia & 1,279 & $28.0 \%$ & 1,056 & $32.7 \%$ & 625 & $31.5 \%$ & 549 & $31.8 \%$ \\
\hline Diabetes & 255 & $5.6 \%$ & 181 & $5.6 \%$ & 114 & $5.7 \%$ & 100 & $5.8 \%$ \\
\hline LT-METs (METs • hrs/day) & 2.72 & 3.69 & 2.84 & 3.31 & 2.49 & 3.18 & 1.96 & 2.68 \\
\hline
\end{tabular}


Table 2. Associations between anamnesis and sedentary time

\begin{tabular}{|c|c|c|c|c|c|c|c|c|}
\hline & \multicolumn{8}{|l|}{ Male } \\
\hline & \multirow[t]{2}{*}{ years } & \multirow[t]{2}{*}{$<5 \mathrm{~h}$} & \multicolumn{2}{|c|}{5 to $<7 \mathrm{~h}$} & \multicolumn{2}{|c|}{7 to $<9 \mathrm{~h}$} & \multicolumn{2}{|c|}{$\geq 9 \mathrm{~h}$} \\
\hline & & & OR & $95 \% \mathrm{CI}$ & OR & $95 \% \mathrm{CI}$ & OR & $95 \% \mathrm{CI}$ \\
\hline \multirow[t]{3}{*}{ Hypertension } & $35-49$ & ref & 1.035 & $0.824-1.301$ & 1.049 & $0.935-1.178$ & 1.036 & $0.973-1.104$ \\
\hline & $50-59$ & ref & 1.071 & $0.925-1.240$ & 1.096 & $1.016-1.182$ & 1.024 & $0.982-1.068$ \\
\hline & $60-69$ & ref & 1.097 & $0.984-1.222$ & 1.011 & $0.952-1.073$ & 1.041 & $1.006-1.077$ \\
\hline \multirow[t]{3}{*}{ Dyslipidemia } & $35-49$ & ref & 1.135 & $0.927-1.389$ & 1.031 & $1.031-1.262$ & 1.122 & $1.061-1.185$ \\
\hline & $50-59$ & ref & 1.377 & $1.175-1.614$ & 1.333 & $1.231-1.444$ & 1.216 & $1.163-1.272$ \\
\hline & $60-69$ & ref & 1.291 & $1.137-1.465$ & 1.166 & $1.088-1.249$ & 1.131 & $1.086-1.177$ \\
\hline \multirow[t]{6}{*}{ Diabetes } & $35-49$ & ref & 0.950 & $0.646-1.397$ & 1.121 & $0.932-1.349$ & 1.011 & $0.910-1.124$ \\
\hline & $50-59$ & ref & 1.103 & $0.881-1.380$ & 1.037 & $0.919-1.169$ & 1.067 & $1.000-1.137$ \\
\hline & $60-69$ & ref & 1.047 & $0.898-1.221$ & 1.071 & $0.986-1.163$ & 1.042 & 0.993-1.093 \\
\hline & \multicolumn{8}{|l|}{ Female } \\
\hline & \multirow[t]{2}{*}{ years } & \multirow[t]{2}{*}{$<5 \mathrm{~h}$} & \multicolumn{2}{|c|}{5 to $<7 \mathrm{~h}$} & \multicolumn{2}{|c|}{7 to $<9 \mathrm{~h}$} & \multicolumn{2}{|c|}{$\geq 9 \mathrm{~h}$} \\
\hline & & & OR & $95 \% \mathrm{CI}$ & OR & $95 \% \mathrm{CI}$ & OR & $95 \% \mathrm{CI}$ \\
\hline \multirow[t]{3}{*}{ Hypertension } & $35-49$ & ref & 0.864 & $0.670-1.114$ & 1.069 & $0.938-1.218$ & 0.980 & $0.901-1.067$ \\
\hline & $50-59$ & ref & 0.999 & $0.875-1.141$ & 0.914 & 0.843-0.992 & 0.951 & $0.902-1.003$ \\
\hline & $60-69$ & ref & 1.041 & $0.938-1.154$ & 1.037 & $0.977-1.102$ & 1.028 & $0.986-1.073$ \\
\hline \multirow[t]{3}{*}{ Dyslipidemia } & $35-49$ & ref & 0.968 & $0.771-1.215$ & 0.965 & $0.848-1.098$ & 1.103 & $1.027-1.184$ \\
\hline & $50-59$ & ref & 1.121 & $0.988-1.272$ & 1.049 & $0.974-1.130$ & 1.083 & $1.033-1.136$ \\
\hline & $60-69$ & ref & 1.252 & $1.134-1.381$ & 1.097 & $1.035-1.162$ & 1.075 & $1.032-1.119$ \\
\hline \multirow[t]{3}{*}{ Diabetes } & $35-49$ & ref & 1.244 & $0.745-2.078$ & 1.120 & $0.842-1.489$ & 1.058 & $0.887-1.263$ \\
\hline & $50-59$ & ref & 0.938 & $0.716-1.230$ & 0.893 & $0.754-1.059$ & 1.024 & $0.926-1.134$ \\
\hline & $60-69$ & ref & 1.007 & $0.827-1.226$ & 1.015 & $0.906-1.138$ & 1.019 & $0.940-1.104$ \\
\hline
\end{tabular}

Adjusted for age, research areas, LT-METs, drinking and smoking status

hypertension, a sedentary time of 7 to $<9$ h/day in the 50-59 years group (OR: 1.096, CI: 1.016-1.182) and in the 60-69 years group (OR: 1.041, CI: 1.006$1.077)$ were associated with a significantly higher proportion of male participants. In female participants, sedentary time was not associated with a higher proportion of hypertension. For dyslipidemia, both male (excluding 5 to $<7 \mathrm{~h} /$ day in the 35-49 years group) and female (excluding 5 to $<7 \mathrm{~h} /$ day and 7 to $<9 \mathrm{~h} /$ day in the 35-49 years group and the 50-59 years group) sexes were associated with a significantly higher proportion of participants with dyslipidemia. For diabetes, sedentary time $\geq 9 \mathrm{~h}$ /day in the 50-59 years group (OR: 1.067, CI: 1.000-1.137) was associated with a significantly higher proportion of male participants. In female participants, sedentary time was not associated with diabetes.

Multiple regression analysis was then performed to identify the variables strongly associated with sed- entary time (Table 3). The lipid cardiometabolic risk factors were significantly associated with sedentary time. As shown in Table 3, sedentary time was significantly associated with TG, HDL-C, and non-HDL-C in males and females. Furthermore, in the 60-69 years group, sedentary time was significantly associated with several variables except HDL-C $(\beta=-0.016, p=$ $0.171)$ as follows: $\operatorname{BMI}(\beta=0.058, p<0.001), \operatorname{SBP}(\beta$ $=0.042, p<0.001)$, DBP $(\beta=0.047, p<0.001), \mathrm{TG}$ $(\beta=0.054, p<0.001)$, non-HDL-C $(\beta=0.039, p=$ $0.001)$, and HbA1c $(\beta=0.024, p=0.044)$. Similarly, sedentary time was significantly associated with BMI $(\beta=0.034, p=0.004)$, SBP $(\beta=0.056, p<0.001)$, DBP $(\beta=0.057, p<0.001)$, TG $(\beta=0.045, p<$ $0.001)$, and HDL-C $(\beta=0.042, p=0.001)$ in obese women. Supplemental Table 1 shows the mean values of BMI, SBP, DBP, TG, HDL-C, non-HDL-C, and $\mathrm{HbA} 1 \mathrm{c}$ levels in each group. Compared with participants who spent $<5 \mathrm{~h}$ /day of sedentary time, those 
Table 3. Comparison of the association of cardiometabolic parameter and sedentary time

\begin{tabular}{|c|c|c|c|c|c|c|}
\hline \multirow[t]{2}{*}{ Male } & \multicolumn{2}{|c|}{$\begin{array}{c}35-49 \text { years } \\
n=4,800\end{array}$} & \multicolumn{2}{|c|}{$\begin{array}{c}50-59 \text { years } \\
n=5,388\end{array}$} & \multicolumn{2}{|c|}{$\begin{array}{c}60-69 \text { years } \\
n=6,921\end{array}$} \\
\hline & beta & $p$-value & beta & $p$-value & beta & $p$-value \\
\hline BMI & 0.072 & 0.072 & 0.015 & 0.278 & 0.058 & $<0.001$ \\
\hline SBP & 0.006 & 0.690 & 0.005 & 0.693 & 0.042 & $<0.001$ \\
\hline DBP & 0.004 & 0.808 & 0.021 & 0.122 & 0.047 & $<0.001$ \\
\hline TG & 0.029 & 0.049 & 0.043 & 0.002 & 0.054 & $<0.001$ \\
\hline HDL-C & -0.049 & 0.001 & -0.054 & $<0.001$ & -0.016 & 0.171 \\
\hline non-HDL-C & 0.031 & 0.034 & 0.038 & 0.006 & 0.039 & 0.001 \\
\hline HbAlc & -0.015 & 0.309 & -0.008 & 0.572 & 0.024 & 0.044 \\
\hline \multirow[t]{2}{*}{ Female } & \multicolumn{2}{|c|}{$\begin{array}{c}35-49 \text { years } \\
n=5,929\end{array}$} & \multicolumn{2}{|c|}{$\begin{array}{c}50-59 \text { years } \\
n=5,986\end{array}$} & \multicolumn{2}{|c|}{$\begin{array}{c}60-69 \text { years } \\
n=6,949\end{array}$} \\
\hline & beta & $p$-value & beta & $p$-value & beta & $p$-value \\
\hline BMI & -0.008 & 0.518 & -0.006 & 0.654 & 0.034 & 0.004 \\
\hline SBP & 0.034 & 0.009 & 0.019 & 0.139 & 0.056 & $<0.001$ \\
\hline DBP & 0.045 & 0.001 & 0.030 & 0.018 & 0.057 & $<0.001$ \\
\hline TG & 0.038 & 0.004 & 0.031 & 0.017 & 0.045 & $<0.001$ \\
\hline HDL-C & 0.020 & 0.127 & 0.007 & 0.610 & 0.042 & 0.001 \\
\hline non-HDL-C & 0.046 & 0.001 & 0.029 & 0.027 & 0.021 & 0.080 \\
\hline HbA1c & -0.036 & 0.006 & -0.040 & 0.002 & -0.003 & 0.815 \\
\hline
\end{tabular}

Adjusted for age, research area, LT-METs, drinking and smoking status

who had longer sedentary time tended to have increased levels of BMI, SBP, DBP, TG, and nonHDL-C and decreased levels of HDL-C, especially in the 60-69 years group. Although sedentary time was not associated with a higher proportion of hypertension among female participants, SBP and DBP tended to increase, resulting from increased sedentary time.

\section{Discussion}

Considerable evidence suggests that sedentary time affects health outcomes regardless of physical activity4. Especially, many previous studies showed that cardiovascular disease and its risk factors are associated with sedentary time ${ }^{3,15-17)}$. This study was conducted to determine the associations of sedentary behavior with cardiometabolic diseases such as hypertension, dyslipidemia, and diabetes mellitus in a large Japanese population according to age and sex.

Among male participants, sedentary time was associated with cardiometabolic diseases. Although sedentary time was not associated with hypertension in female participants, Sedentary time increased; thus, SBP and DBP tended to increase. These results suggest the association between sedentary time and cardiometabolic diseases in Japanese males and females.
In agreement with many previous studies, our study confirmed that sedentary time was associated with cardiometabolic diseases $\left.{ }^{2}, 3,16,18-21\right)$. Notably, our results further revealed that sedentary time was strongly associated with dyslipidemia and lipid metabolism, as indicated by the levels of TG, HDL-C, and nonHDL-C. A recent study showed the mechanism of adverse effects of sedentary time; that is, the sedentary time has a potential role in the increased production of reactive oxygen species, low-grade inflammation, and metabolic impairment, which contribute to sitting-induced impaired vascular function ${ }^{1)}$. Furthermore, inactivity, such as sitting, quickly engages signals for specific molecular responses contributing to poor lipid metabolism by suppression of skeletal muscle lipoprotein lipase (LPL ${ }^{22)}$; a protein important for controlling plasma TG catabolism, HDL-C, and other metabolic risk factors) activity ${ }^{23)}$. LPL activity was associated with reduced TG uptake and decreased HDL-C levels ${ }^{24)}$. In contrast, the response of lipids and lipoproteins was improved by physical activity, including regular aquatic endurance ${ }^{25)}$, cardiorespiratory exercise ${ }^{26)}$, and fitness aerobic exercise ${ }^{27)}$. These studies show that sedentary time and physical activity has a significant effect on lipid metabolism, which is consistent with our results. The findings of the present 
study partly support these previous results. To the best of our knowledge, this is the first study to show that sedentary time was strongly associated with lipid metabolism in a large Japanese population.

From the analysis of continuous variables, compared with female participants who had $<5$ h per day of sedentary time, those who had $\geq 9$ h of sedentary time per day in the 60-69 years group had $3.4 \mathrm{mmHg}$ higher SBP. In The Japanese Society of Hypertension Guidelines for the Management of Hypertension, the cardiovascular disease-reducing effects of lowering blood pressure were estimated using the EPOCHJAPAN database ${ }^{28)}$. Briefly, a decrease of only 4 $\mathrm{mmHg}$ in the average SBP in the Japanese population is estimated to reduce the age-adjusted mortality from stroke in males and females by $8.9 \%$ and $5.8 \%$, respectively (the total number of deaths from stroke will decrease by 10,000 per year) and that for coronary heart disease by $5.4 \%$ and $7.2 \%$, respectively (the total number of deaths from coronary heart disease will decrease by 5,000 per year ${ }^{28)}$. This may indicate that reducing sedentary time was an effective strategy to lower the blood pressure of the population. Furthermore, one study indicated that replacing sedentary time with the same amount of moderate- to vigorousintensity physical activity may contribute to better physical health-related quality of life of Japanese older adults ${ }^{29)}$. In summary, regularly interrupting and replacing sedentary time may contribute to better health-related quality of life because of population approach for the prevention of cardiometabolic diseases.

In contrast, $\mathrm{HbA} 1 \mathrm{c}$ tended to decrease, in the 35-49 years females group and the 50-59 years both sexes group. The impact of sedentary time on glycaemic biomarkers was limited in a systematic review ${ }^{30}$. It seems difficult to evaluate the relationship between sitting time and $\mathrm{HbAlc}$ in the general population ${ }^{31)}$ or to use HbA1c as an indicator of glucose metabolism ${ }^{32)}$. To clarify the relationship between glycemic biomarkers and sedentary time, further study and/or more sensitive measures of insulin resistance are necessary.

Despite our novel findings, this study has some limitations. This study uses a cross-sectional design and self-administered questionnaires to evaluate sedentary time and anamnesis. Although questionnaire evaluation of sitting time is controversial, IPAQ was an acceptable international physical activity surveillance instrument ${ }^{33)}$. Most of the studies about sedentary time made use of self-reported sedentary behaviors ${ }^{19)}$. Furthermore, a previous study reported that both accelerometer and self-report measurements are similarly associated with cardiometabolic risk factors in the Japanese population ${ }^{34)}$. Similarly, a previous study reported that stroke and myocardial infarction appears sensitive enough to be used for baseline evaluation of patient characteristics in Japanese cohort studies $^{35)}$. In a follow-up survey, we plan to assess the participants using their actual medical records; therefore, we expect that these additional data will help with further detailed analysis of the direct effect of sedentary time. The strength of the study is that a large number of participants were included, and we implemented a population-based cohort design.

\section{Conclusion}

Independent of leisure-time physical activity, sedentary time was associated with cardiometabolic diseases in a large Japanese population classified by age and sex. Our findings indicate that regularly interrupting and replacing sedentary time may contribute to better health-related quality of life because of population approach for the prevention of cardiometabolic diseases.

\section{Financial Support}

This study was funded by Grants-in-Aid for Scientific Research on Priority Areas of Cancer (No. 17015018) and on Innovative Areas (No. 221S0001), Platform of Supporting Cohort Study and Biospecimen Analysis (JSPS KAKENHI Grant Number JP16H06277) from the Japanese Ministry of Education, Culture, Sports, Science and Technology, and by Grants-in-Aid for Scientific Research (C) (JSPS KAKENHI Grant Number JP15K08778 and JP18K10086) from the Japan Society for the Promotion of Science.

\section{Conflict of Interest}

The authors declare that they have no conflict of interest.

\section{References}

1) Carter S, Hartman Y, Holder S, Thijssen DH and Hopkins ND: Sedentary Behavior and Cardiovascular Disease Risk: Mediating Mechanisms. Exerc Sport Sci Rev, 2017; 45: 80-86

2) Young DR, Hivert MF, Alhassan S, Camhi SM, Ferguson JF, Katzmarzyk PT, Lewis CE, Owen N, Perry CK, Siddique J and Yong CM: Sedentary Behavior and Cardiovascular Morbidity and Mortality: A Science Advisory From the American Heart Association. Circulation, 2016; 134: e262-279 
3) Wilmot EG, Edwardson CL, Achana FA, Davies MJ, Gorely T, Gray LJ, Khunti K, Yates T and Biddle SJ: Sedentary time in adults and the association with diabetes, cardiovascular disease and death: systematic review and meta-analysis. Diabetologia, 2012; 55: 2895-2905

4) Biswas A, Oh PI, Faulkner GE, Bajaj RR, Silver MA, Mitchell MS and Alter DA: Sedentary time and its association with risk for disease incidence, mortality, and hospitalization in adults: a systematic review and meta-analysis. Ann Intern Med, 2015; 162: 123-132

5) Copeland JL, Ashe MC, Biddle SJ, Brown WJ, Buman MP, Chastin S, Gardiner PA, Inoue S, Jefferis BJ, Oka K, Owen N, Sardinha LB, Skelton DA, Sugiyama T and Dogra S: Sedentary time in older adults: a critical review of measurement, associations with health, and interventions. Br J Sports Med, 2017; 51: 1539

6) Furukawa $S$, Sakai T, Niiya T, Miyaoka $H$, Miyake $T$, Yamamoto S, Kanzaki S, Maruyama K, Tanaka K, Ueda T, Senba H, Torisu M, Minami H, Tanigawa T, Matsuura B, Hiasa Y and Miyake Y: Self-reported sitting time and prevalence of erectile dysfunction in Japanese patients with type 2 diabetes mellitus: The Dogo Study. J Diabetes Complications, 2017; 31: 53-57

7) Lee $S$, Shimada H, Lee $S$, Makizako H, Doi T, Harada K, Bae S, Harada K, Hotta R, Tsutsumimoto K, Yoshida D, Nakakubo S, Anan Y, Park H and Suzuki T: Association between sedentary time and kidney function in community-dwelling elderly Japanese people. Geriatr Gerontol Int, 2017; 17: 730-736

8) Ukawa S, Tamakoshi A, Yatsuya H, Yamagishi K, Ando $\mathrm{M}$ and Iso H: Association Between Average Daily Television Viewing Time and Chronic Obstructive Pulmonary Disease-Related Mortality: Findings From the Japan Collaborative Cohort Study. J Epidemiol, 2015; 25: 431-436

9) Ikehara $S$, Iso H, Wada Y, Tanabe N, Watanabe Y, Kikuchi $S$ and Tamakoshi A: Television viewing time and mortality from stroke and coronary artery disease among Japanese men and women -- the Japan Collaborative Cohort Study. Circ J, 2015; 79: 2389-2395

10) Kikuchi H, Inoue $S$, Odagiri $Y$, Inoue $M$, Sawada $N$ and Tsugane S: Occupational sitting time and risk of all-cause mortality among Japanese workers. Scand J Work Environ Health, 2015; 41: 519-528

11) Hamajima N: The Japan Multi-Institutional Collaborative Cohort Study (J-MICC Study) to detect gene-environment interactions for cancer. Asian Pac J Cancer Prev, 2007; 8: 317-323

12) Craig CL, Marshall AL, Sjostrom M, Bauman AE, Booth ML, Ainsworth BE, Pratt M, Ekelund U, Yngve A, Sallis JF and Oja P: International physical activity questionnaire: 12-country reliability and validity. Med Sci Sports Exerc, 2003; 35: 1381-1395

13) Hara M, Higaki Y, Taguchi N, Shinchi K, Morita E, Naito M, Hamajima N, Takashima N, Suzuki S, Nakamura A, Ohnaka K, Uemura H, Nishida H, Hosono S, Mikami H, Kubo M, Tanaka H and Japan Multi-Institutional Collaborative Cohort Study G: Effect of the PPARG2 Pro12Ala polymorphism and clinical risk factors for diabetes mellitus on HbA1c in the Japanese general population. J Epidemiol, 2012; 22: 523-531

14) Hara M, Hachiya T, Sutoh Y, Matsuo K, Nishida Y, Shi- manoe C, Tanaka K, Shimizu A, Ohnaka K, Kawaguchi T, Oze I, Matsuda F, Ito H, Kawai S, Hishida A, Okada R, Sasakabe T, Hirata A, Ibusuki R, Nindita Y, Furusyo N, Ikezaki H, Kuriyama N, Ozaki E, Mikami H, Nakamura Y, Suzuki S, Hosono A, Katsuura-Kamano S, Arisawa K, Kuriki K, Endoh K, Takashima N, Kadota A, Nakatochi M, Momozawa Y, Kubo M, Naito M and Wakai K: Genomewide Association Study of Leisure-Time Exercise Behavior in Japanese Adults. Med Sci Sports Exerc, 2018; 50: 2433-2441

15) Ekelund U, Steene-Johannessen J, Brown WJ, Fagerland MW, Owen N, Powell KE, Bauman A and Lee IM: Does physical activity attenuate, or even eliminate, the detrimental association of sitting time with mortality? A harmonised meta-analysis of data from more than 1 million men and women. Lancet, 2016; 388: 1302-1310

16) Patterson R, McNamara E, Tainio M, de Sa TH, Smith AD, Sharp SJ, Edwards P, Woodcock J, Brage S and Wijndaele K: Sedentary behaviour and risk of all-cause, cardiovascular and cancer mortality, and incident type 2 diabetes: a systematic review and dose response meta-analysis. Eur J Epidemiol, 2018; 33: 811-829

17) Powell C, Herring MP, Dowd KP, Donnelly AE and Carson BP: The cross-sectional associations between objectively measured sedentary time and cardiometabolic health markers in adults - a systematic review with metaanalysis component. Obes Rev, 2018; 19: 381-395

18) Lim MS, Park B, Kong IG, Sim S, Kim SY, Kim JH and Choi HG: Leisure sedentary time is differentially associated with hypertension, diabetes mellitus, and hyperlipidemia depending on occupation. BMC Public Health, 2017; 17: 278

19) Lee PH and Wong FK: The association between time spent in sedentary behaviors and blood pressure: a systematic review and meta-analysis. Sports Med, 2015; 45: 867 880

20) Ford ES and Caspersen CJ: Sedentary behaviour and cardiovascular disease: a review of prospective studies. Int J Epidemiol, 2012; 41: 1338-1353

21) Same RV, Feldman DI, Shah N, Martin SS, Al Rifai M, Blaha MJ, Graham G and Ahmed HM: Relationship Between Sedentary Behavior and Cardiovascular Risk. Curr Cardiol Rep, 2016; 18: 6

22) Olivecrona G: Role of lipoprotein lipase in lipid metabolism. Curr Opin Lipidol, 2016; 27: 233-241

23) Hamilton MT, Hamilton DG and Zderic TW: Role of low energy expenditure and sitting in obesity, metabolic syndrome, type 2 diabetes, and cardiovascular disease. Diabetes, 2007; 56: 2655-2667

24) Bey L and Hamilton MT: Suppression of skeletal muscle lipoprotein lipase activity during physical inactivity: a molecular reason to maintain daily low-intensity activity. J Physiol, 2003; 551: 673-682

25) Igarashi $Y$ and Nogami Y: Response of Lipids and Lipoproteins to Regular Aquatic Endurance Exercise: A MetaAnalysis of Randomized Controlled Trials. J Atheroscler Thromb, 2019; 26: 14-30

26) Watanabe N, S SS, Shimada K, Lee IM, Gando Y, Momma H, Kawakami R, Miyachi M, Hagi Y, Kinugawa C, Okamoto T, Tsukamoto K and S NB: Relationship between Cardiorespiratory Fitness and Non-High-Density 
Lipoprotein Cholesterol: A Cohort Study. J Atheroscler Thromb, 2018; 25: 1196-1205

27) Igarashi $Y$, Akazawa $N$ and Maeda S: Effects of Aerobic Exercise Alone on Lipids in Healthy East Asians: A Systematic Review and Meta-Analysis. J Atheroscler Thromb, 2019; 26: 488-503

28) Shimamoto K, Ando K, Fujita T, Hasebe N, Higaki J, Horiuchi M, Imai Y, Imaizumi T, Ishimitsu T, Ito M, Ito $\mathrm{S}$, Itoh $\mathrm{H}$, Iwao $\mathrm{H}$, Kai $\mathrm{H}$, Kario $\mathrm{K}$, Kashihara $\mathrm{N}$, Kawano Y, Kim-Mitsuyama S, Kimura G, Kohara K, Komuro I, Kumagai H, Matsuura H, Miura K, Morishita R, Naruse M, Node K, Ohya Y, Rakugi H, Saito I, Saitoh S, Shimada K, Shimosawa T, Suzuki H, Tamura K, Tanahashi N, Tsuchihashi T, Uchiyama M, Ueda $S$ and Umemura S: The Japanese Society of Hypertension Guidelines for the Management of Hypertension (JSH 2014). Hypertens Res, 2014; 37: 253-390

29) Yasunaga $A$, Shibata $A$, Ishii $K$, Inoue $S$, Sugiyama $T$, Owen $\mathrm{N}$ and Oka K: Replacing sedentary time with physical activity: effects on health-related quality of life in older Japanese adults. Health Qual Life Outcomes, 2018; 16: 240

30) Wirth K, Klenk J, Brefka S, Dallmeier D, Faehling K, Roque IFM, Tully MA, Gine-Garriga M, Caserotti P, Salva A, Rothenbacher D, Denkinger M and Stubbs B: Biomarkers associated with sedentary behaviour in older adults: A systematic review. Ageing Res Rev, 2017; 35:
$87-111$

31) Bellettiere J, Winkler EAH, Chastin SFM, Kerr J, Owen N, Dunstan DW and Healy GN: Associations of sitting accumulation patterns with cardio-metabolic risk biomarkers in Australian adults. PLoS One, 2017; 12: e0180119

32) Henson J, Yates T, Biddle SJ, Edwardson CL, Khunti K, Wilmot EG, Gray LJ, Gorely T, Nimmo MA and Davies MJ: Associations of objectively measured sedentary behaviour and physical activity with markers of cardiometabolic health. Diabetologia, 2013; 56: 1012-1020

33) Bauman A, Bull F, Chey T, Craig CL, Ainsworth BE, Sallis JF, Bowles HR, Hagstromer M, Sjostrom M and Pratt M: The International Prevalence Study on Physical Activity: results from 20 countries. Int J Behav Nutr Phys Act, 2009; 6: 21

34) Honda T, Chen $S$, Kishimoto $H$, Narazaki $K$ and Kumagai S: Identifying associations between sedentary time and cardio-metabolic risk factors in working adults using objective and subjective measures: a cross-sectional analysis. BMC Public Health, 2014; 14: 1307

35) Yamagishi $K$, Ikeda $A$, Iso $H$, Inoue $M$ and Tsugane $S$ : Self-reported stroke and myocardial infarction had adequate sensitivity in a population-based prospective study JPHC (Japan Public Health Center)-based Prospective Study. Journal of clinical epidemiology, 2009; 62: 667-673 
Supplemental Table 1. The mean values of BMI, SBP, DBP, TG, HDL-C, nonHDL-C and HbA1c levels in each sedentary time group

\begin{tabular}{|c|c|c|c|c|c|c|c|c|c|c|}
\hline \multicolumn{11}{|l|}{ Male } \\
\hline years & & \multicolumn{2}{|c|}{$\begin{array}{c}<5 \mathrm{~h} \\
n=6,077\end{array}$} & \multicolumn{2}{|c|}{$\begin{array}{c}5 \text { to }<7 \mathrm{~h} \\
n=3,440\end{array}$} & \multicolumn{2}{|c|}{$\begin{array}{c}7 \text { to }<9 \text { h } \\
n=2,755\end{array}$} & \multicolumn{2}{|c|}{$\begin{aligned} & \geq 9 \mathrm{~h} \\
n & =4,837\end{aligned}$} & $p$ trend \\
\hline \multirow[t]{5}{*}{$35-49$} & BMI $\left(\mathrm{kg} / \mathrm{m}^{2}\right)$ & 23.76 & 3.34 & 23.66 & 3.18 & 23.90 & 3.38 & 23.89 & 3.33 & 0.176 \\
\hline & SBP (mmHg) & 122.8 & 16.02 & 122.7 & 15.89 & 122.6 & 15.77 & 123.9 & 15.84 & 0.017 \\
\hline & HDL-C (mg/dl) & 57.94 & 15.26 & 57.14 & 14.24 & 56.31 & 15.41 & 56.25 & 14.24 & 0.002 \\
\hline & non-HDL-C (mg/dl) & 145.3 & 35.35 & 145.6 & 37.74 & 144.7 & 33.53 & 148.5 & 35.18 & 0.005 \\
\hline & HbA1c (\%) & 5.47 & 0.63 & 5.41 & 0.55 & 5.48 & 0.71 & 5.41 & 0.60 & $<0.001$ \\
\hline \multirow[t]{4}{*}{$50-59$} & BMI $\left(\mathrm{kg} / \mathrm{m}^{2}\right)$ & 23.68 & 2.98 & 23.87 & 3.11 & 23.84 & 2.92 & 23.82 & 2.98 & 0.151 \\
\hline & SBP $(\mathrm{mmHg})$ & 129.3 & 18.21 & 128.5 & 18.20 & 130.1 & 18.83 & 130.0 & 17.98 & 0.114 \\
\hline & non-HDL-C (mg/dl) & 146.1 & 33.98 & 150.7 & 33.73 & 150.4 & 35.55 & 150.0 & 33.78 & 0.001 \\
\hline & HbA1c (\%) & 5.66 & 0.75 & 5.71 & 0.90 & 5.67 & 0.75 & 5.61 & 0.72 & $<0.001$ \\
\hline \multirow[t]{7}{*}{$60-69$} & BMI $\left(\mathrm{kg} / \mathrm{m}^{2}\right)$ & 23.22 & 2.71 & 23.29 & 2.73 & 23.42 & 2.85 & 23.66 & 2.93 & $<0.001$ \\
\hline & $\mathrm{SBP}(\mathrm{mmHg})$ & 133.6 & 18.71 & 135.3 & 19.37 & 135.6 & 19.43 & 135.6 & 19.40 & $<0.001$ \\
\hline & DBP (mmHg) & 80.94 & 10.74 & 81.87 & 10.93 & 82.22 & 10.76 & 82.16 & 11.04 & $<0.001$ \\
\hline & $\mathrm{TG}(\mathrm{mg} / \mathrm{dl})$ & 127.8 & 88.90 & 136.3 & 97.06 & 139.5 & 92.21 & 142.1 & 95.58 & $<0.001$ \\
\hline & HDL-C (mg/dl) & 59.49 & 15.98 & 58.96 & 15.52 & 58.13 & 15.74 & 58.16 & 15.66 & 0.002 \\
\hline & non-HDL-C (mg/dl) & 142.8 & 32.27 & 144.4 & 32.49 & 146.1 & 33.83 & 146.1 & 34.62 & 0.001 \\
\hline & HbAlc (\%) & 5.71 & 0.74 & 5.71 & 0.72 & 5.73 & 0.69 & 5.74 & 0.76 & 0.370 \\
\hline
\end{tabular}

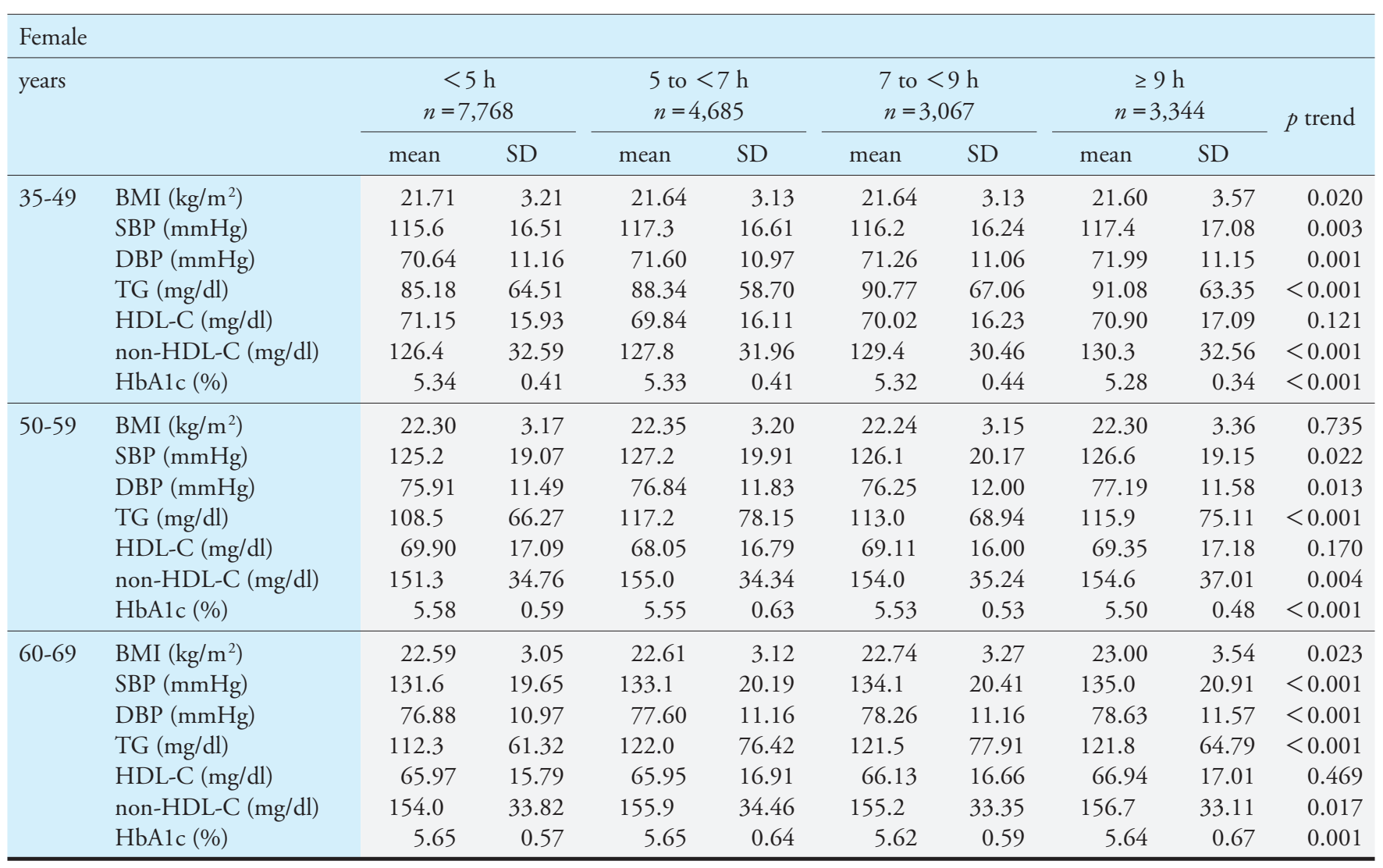

